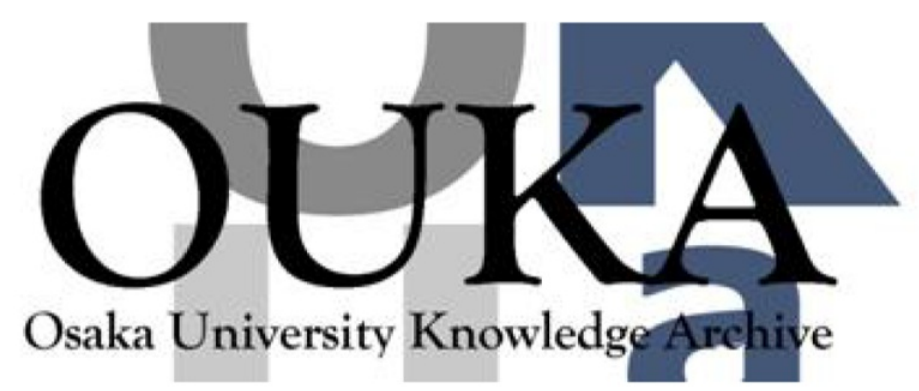

\begin{tabular}{|c|l|}
\hline Title & $\begin{array}{l}\text { Log Hodge theoretic formulation of mirror } \\
\text { symmetry for Calabi-Yau threefolds }\end{array}$ \\
\hline Author(s) & Usui, Sampe i \\
\hline Citation & $\begin{array}{l}\text { Vietnam Journal of Mathematics. 42(3) p. 345- } \\
\text { p. } 363\end{array}$ \\
\hline Issue Date & 2014 \\
\hline oaire:version & AM \\
\hline URL & https://hdl. handle. net/11094/73430 \\
\hline rights & \\
\hline Note & \\
\hline
\end{tabular}

Osaka University Knowledge Archive : OUKA

https://ir. Library. osaka-u. ac. jp/

Osaka University 


\title{
LOG HODGE THEORETIC FORMULATION OF MIRROR SYMMETRY FOR CALABI-YAU THREEFOLDS
}

\author{
SAMPEI USUI ${ }^{1}$
}

\author{
Dedicated to Professor Hideyasu Sumihiro
}

\begin{abstract}
We hope to understand the Hodge theoretic aspect of mirror symmetry in the framework of the fundamental diagram of log mixed Hodge theory. We give a formulation of mirror conjecture for Calabi-Yau threefolds as the coincidence of log period maps with specified sections under the mirror map. Since a variation of Hodge structure with unipotent monodromies on a product of punctured discs uniquely extends over the puncture to a log Hodge structure, we can work on the boundary point over which the log Riemann-Hilbert correspondence exists, and we can observe clearly in high-resolution the behavior of $\mathbf{Z}$-structure over the boundary point, cf. Notes in Introduction below. This is an advantage of log Hodge theory.
\end{abstract}

\section{Contents}

Introduction

1. Log Hodge theory

1.1. Category $\mathcal{B}(\log )$

1.2. Ringed space $\left(S^{\log }, \mathcal{O}_{S}^{\log }\right)$

1.3. Polarized log Hodge structure

1.4. Nilpotent orbit and period map

1.5. Toric variety

1.6. Moduli of polarized log Hodge structures of specified type

2. Calabi-Yau threefolds

2.1. Picard-Fuchs ideal

2.2. B-model of $V^{\circ}$

2.3. A-model of $V$

2.4. Z-structure

2.5. Correspondence table

2.6. On equivalence of formulations (1)-(4) in Introduction References

2000 Mathematics Subject Classification. Primary 14C30; Secondary 14D07, 32G20.

${ }^{1}$ Partially supported by JSPS KAKENHI (B) No. 23340008. 


\section{Introduction}

In this paper, a Calabi-Yau threefold is assumed to be simply connected.

Assume that $V$ and $V^{\circ}$ are smooth Calabi-Yau threefolds which are mirrors. After [CK99], we also impose the following optimistic assumptions:

- A maximally unipotent monodromy (MUM, for short) point $p_{0}$ lying on a smooth compactification $\overline{\mathcal{M}}\left(V^{\circ}\right)$ of the complex moduli space of $V^{\circ}$ (cf. loc.cit., Definition 5.2.2).

- A large radius limit point $q_{0}$ lying on a compatible smooth compactification $\overline{\mathcal{K} \mathcal{M}}(V)$ of the Kähler moduli space of $V$ (cf. loc.cit., Section 8.5.2).

- The mirror map, by the canonical coordinates $q_{1}, \ldots, q_{r}$ from Sections 2.2 and 2.3 below, maps a neighborhood of $p_{0} \in \overline{\mathcal{M}}\left(V^{\circ}\right)$ to a neighborhood of $q_{0} \in$ $\overline{\mathcal{K} \mathcal{M}}(V)$ (cf. loc.cit., Section 6.3.1).

We will assume that good choices of $p_{0}$ and $q_{0}$ have been made, and that $V^{\circ}$ satisfies Integrality Conjecture in [M97], [CK99, Definition 5.2.2], which specifies the mirror map uniquely ([M97], [CK99, Section 6.3.1]). Let $t_{j}:=y_{j} / y_{0}, u_{j}:=t_{j} / 2 \pi i$ and $q_{j}:=e^{t_{j}}=$ $e^{2 \pi i u_{j}}(1 \leq j \leq r)$ from Section 2.2 below.

The following formulations (1)-(3) of mirror symmetry conjecture are well-known.

(1) (Potential) A-model potential $\Phi_{V}^{\mathrm{GW}}$ for $V$ from Section 2.3 and B-model potential $\Phi_{V^{\circ}}^{\mathrm{GM}}$ for $V^{\circ}$ from Section 2.2 coincide

$$
\Phi_{V}^{\mathrm{GW}}\left(t_{1}, \ldots, t_{r}\right)=\Phi_{V^{\circ}}^{\mathrm{GM}}\left(t_{1}, \ldots, t_{r}\right)
$$

up to quadratic terms.

(2) (Yukawa Coupling) The B-model correlation functions $Y_{i j k}$ of $V^{\circ}$ and the A-model correlation functions $\left\langle T_{i}, T_{j}, T_{k}\right\rangle$ of $V$ satisfy

$$
Y_{i j k}=\left\langle T_{i}, T_{j}, T_{k}\right\rangle \quad(1 \leq i, j, k \leq r) .
$$

(3) (Variation of Hodge structure. [M97], cf. [CK99, Definition 8.6.1]) The isomorphism $\left(q_{0} \in \overline{\mathcal{K} \mathcal{M}}(V)\right) \leftarrow\left(p_{0} \in \overline{\mathcal{M}}\left(V^{\circ}\right)\right)$ of neighborhoods of the compactifications, given by the canonical coordinates $q_{j}=\exp \left(2 \pi i u_{j}\right)(1 \leq j \leq r)$, lifts to an isomorphism

$$
\left(\mathcal{H}^{V}, S, \nabla^{\text {middle }}, \mathcal{H}_{\mathbf{Z}}^{V}, \mathcal{F}, 1,[\mathrm{pt}]\right) \stackrel{\sim}{\leftarrow}\left(\mathcal{H}^{V^{\circ}}, Q, \nabla^{\mathrm{GM}}, \mathcal{H}_{\mathbf{Z}}^{V^{\circ}}, \mathcal{F}, \tilde{\Omega}, g_{0}\right)
$$

over $\mathcal{K} \mathcal{M}(V) \stackrel{\mathcal{M}}{\leftarrow}\left(V^{\circ}\right)$ of the B-variation of polarized Hodge structure with specified sections $\tilde{\Omega}$ and $g_{0}$ for $V^{\circ}$ (from 2.2 and 2.5 (2B) below) and the A-variation of polarized Hodge structure with specified sections 1 and [pt] for $V$.

In this paper, we give another formulation (4) below. The equivalence between (4) and (1)-(3) follows immediately by a log Hodge theoretic interpretation [KU09, Theorem 2.14] of nilpotent orbit theorem of Schmid [S73] (see Proof 1 in 2.6 below). We give another plain proof, Proof 2 in 2.6 below, which is based on the correspondence table in 2.5 below. 
(4) (Log variation of Hodge structure, i.e., Log period map) The isomorphism $\left(q_{0} \in\right.$ $\overline{\mathcal{K} \mathcal{M}}(V)) \stackrel{\leftarrow}{\leftarrow}\left(p_{0} \in \overline{\mathcal{M}}\left(V^{\circ}\right)\right)$ of neighborhoods of the compactifications, given by the canonical coordinates $q_{j}=\exp \left(2 \pi i u_{j}\right)(1 \leq j \leq r)$, lifts to an isomorphism of the B-log variation of polarized Hodge structure with specified sections $\tilde{\Omega}$ and $g_{0}$ and the A-log variation of polarized Hodge structure with specified sections 1 and [pt] over $\overline{\mathcal{K} \mathcal{M}}(V) \leftarrow \overline{\mathcal{M}}\left(V^{\circ}\right)$, whose restriction to the punctured $\mathcal{K} \mathcal{M}(V) \leftarrow \mathcal{M}\left(V^{\circ}\right)$ yields the isomorphism of variations of polarized Hodge structure with specified sections in (3).

This rephrases as follows: After identifying symplectic bases of the reference fibers of the local system over $\mathbf{Z}$, we have a commutative diagram of horizontal log period maps

$$
\begin{gathered}
\left(q_{0} \in \overline{\mathcal{K} \mathcal{M}}(V)\right) \stackrel{\leftarrow}{\leftarrow}\left(p_{0} \in \overline{\mathcal{M}}\left(V^{\circ}\right)\right) \\
\searrow \quad \swarrow \\
\left(\left[\sigma, \exp \left(\sigma_{\mathbf{C}}\right) F_{0}\right] \in \Gamma \backslash D_{\sigma}\right)
\end{gathered}
$$

with extensions of specified sections in (3). Here $\sigma$ is the cone generated by the logarithms of local monodromies and $\Gamma$ is the group generated by the local monodromies both of which are transformed by the level structure into End and Aut, respectively, of the reference fiber of the local system, and $\left(\sigma, \exp \left(\sigma_{\mathbf{C}}\right) F_{0}\right)$ is the nilpotent orbit and $\Gamma \backslash D_{\sigma}$ is the fine moduli of log Hodge structures of specified type (1.6 below).

Let $D$ be a classifying space $\mathrm{D}$ of Hodge structures of specified type. In the series of joint works [KU99], [KU02], and [KU09], we constructed various kind of extensions of $D$ and showed their relation as the following diagram:

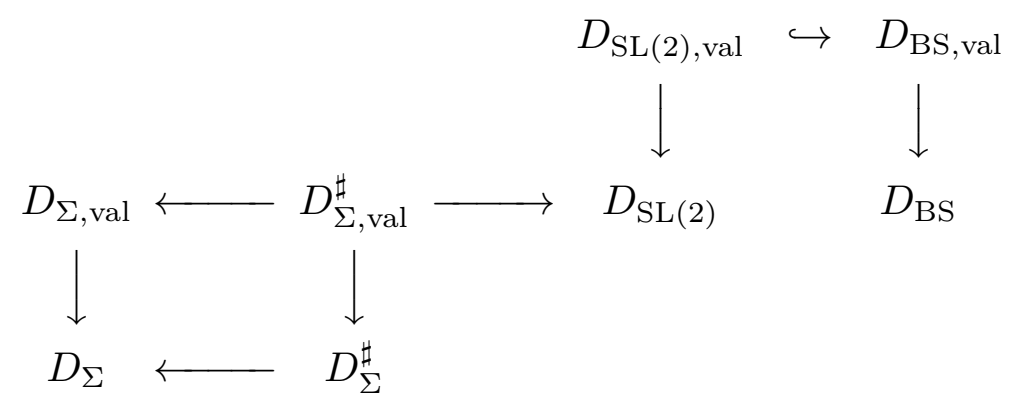

We call this Fundamental Diagram, because this yields a global framework. Indeed, this diagram, taken the quotients by a discrete group $\Gamma$, shows the relation of various kind of compactifications of $\Gamma \backslash D$, i.e., shows the relation of various kind of limits of a series of points of $\Gamma \backslash D$. We hope to observe Hodge theoretic side of mirror symmetry in Fundamental Diagram. The above formulation (4), which is local, is a first step.

In Section 1, we give a brief guide to log Hodge theory from [KU09] for the reader's convenience, which will be used in the proof of the equivalence of the formulation (4) with the other ones (1)-(3). In Section 2, we review mirror symmetry conjecture of Calabi-Yau threefolds from [CK99], then we extend variations of Hodge structures from the formulation (3) over the limit points. Especially, we clarify extension of $\mathbf{Z}$ structures by using log Hodge theory, and prove the formulation (4) is equivalent to the other formulations (1)-(3). 
Notes added in revision. The author received important information and useful comments from a referee. These notes are added as answers to them.

(i) For a Batyrev's mirror pair of Calabi-Yau hypersurfaces, H. Iritani defined ambiant $\mathbf{Z}$-structure of $A$-model of the hypersurface and residual (i.e., vanishing cycle) $\mathbf{Z}$ structure of $B$-model on the mirror, and proved that they are isomorphic as $\mathbf{Z}$-variations of Hodge structure (Theorem 6.9 in [I11]). For quintic threefold and its mirror, he also showed that the above isomorphism extends to the isomorphism between their overlattices induced from integral cohomologies (Example 6.18, loc. cit.).

(ii) As Proof 1 in 2.6 of the present paper, the correspondence table in 2.5 is not necessary for a proof of the equivalence of the formulation (4) with (1)-(3). But, the correspondence table is useful to formulate suitably the domainwall tension in open mirror symmetry for quintic threefolds and to investigate it in the framework of Néron model for admissible normal function constructed in the joint work [KNU14] with K. Kato and C. Nakayama. In this way, we proved the following assertions: In a neighborhood of MUM point in the $B$-model, the domainwall tension determines uniquely a normal function (i.e., an element $H$ of $\mathcal{E} x t^{1}\left(\mathbf{Z}, \mathcal{H}_{\mathbf{z}}\right)$ ) in the category of log mixed Hodge structures, and the inverse of this normal function from its image is given by $16 \pi^{2} / 15$ times the Picard-Fuchs differential operator applying to the extension of the local system $H_{\mathbf{Q}}$; The mirror of the integral relative cohomology sequence of quintic threefold and a real Lagrangian in $A$-model side is given in $B$-model side of the mirror by the difference of a lifting $1_{\mathbf{Z}}$ of $1 \in \mathbf{Z}$ to the local system $H_{\mathbf{Z}}$ and its split modification $1_{\mathbf{Z}}^{\mathrm{spl}}$. Moreover, in this case, it is shown that the classical contribution is computed on the maximal unipotent monodromy point endowed with the log structure induced from the one associated to the boundary divisor on the parameter space.

(iii) As in Acknowledgements below, H. Iritani pointed out the computations of flat integral bases in [U13p] were insufficient. They are corrected in [U14p].

Acknowledgements. The author would like to thank Kazuya Kato and Chikara Nakayama for exciting collaborations and useful comments. The author would like to thank the referees for important information and helpful comments. The author would like to thank Hiroshi Iritani who pointed out that the treatment of $\mathbf{Z}$-structure in Sections 2.4 and 2.5 in old version was insuffcient.

\section{Log Hodge theory}

We recall here, for the reader's convenience, the minimum size of the results of log Hodge theory from [KU09], which will be used in this paper.

\subsection{Category $\mathcal{B}(\log )$}

Let $Z$ be an analytic space and $S$ be a subset of $Z$. A subset $U \subset S$ is open in the strong topology of $S$ in $Z$ if, for any analytic space $A$ and any morphism $f: A \rightarrow Z$ of analytic spaces satisfying $f(A) \subset S$ as sets, $f^{-1}(U)$ is open in $A$. A subset $S$, endowed with the strong topology of $S$ in $Z$ and with the induced sheaf of local rings, is called a strong subspace. 
For a local ringed space $S$, a $\log$ structure on $S$ is a sheaf of monoids $M$ on $S$ together with a monoid homomorphisim $\alpha: M \rightarrow \mathcal{O}_{S}$ satisfying $\alpha^{-1} \mathcal{O}_{S}^{\times} \stackrel{\sim}{\rightarrow} \mathcal{O}_{S}^{\times}$. Here $\mathcal{O}_{S}$ is viewed as a sheaf of monoids by multiplication. An fs log structure is a log structure which satisfies the condition "fs", meaning finitely generated, integral and saturated (for the precise definition, see e.g. [KU09, Section 2.1.5]).

An analytic space endowed with an fs log structure is called an fs log analytic space. We denote by $\mathcal{B}(\log )$ the category of local ringed spaces $S$ over $\mathbf{C}$ endowed with fs log structure such that, locally, $S$ is isomorphic to a strong subspace of an fs log analytic space.

An fs log analytic space is called log smooth if, locally, it is isomorphic to an open set of a toric variety. A smooth object of $\mathcal{B}(\log )$ is called a log manifold, which is, roughly speaking, a $\log$ smooth fs log analytic space allowing slits and endowed with strong topology. For precise definition, see e.g. [KU09, Definition 3.5.7]. A typical example of a slit is as follows:

Example. $S:=((\mathbf{C} \times \mathbf{C}) \backslash(\{0\} \times \mathbf{C})) \cup\{(0,0)\} \subset Z:=\mathbf{C}^{2}$.

\subsection{Ringed space $\left(S^{\log }, \mathcal{O}_{S}^{\log }\right)$}

Let $S$ be an object of $\mathcal{B}(\log )$.

As a set, we define

$$
S^{\log }:=\left\{(s, h) \mid s \in S, h: M_{s}^{\mathrm{gp}} \rightarrow \mathbf{S}^{1} \text { homomorphism s.t. } h(u)=u /|u|\left(u \in \mathcal{O}_{S, s}^{\times}\right)\right\} .
$$

Here $M^{\text {gp }}$ is the sheaf of abelian groups associated to $M$ (i.e., fractions of $M$ ), and $\mathbf{S}^{1}$ is the unit circle in $\mathbf{C}$. We endow $S^{\log }$ with the weakest topology for which the following maps (1) and (2) are continuous.

(1) $\tau: S^{\log } \rightarrow S,(s, h) \mapsto s$.

(2) For any open $U \subset S$ and any $f \in \Gamma\left(U, M^{\mathrm{gp}}\right), \tau^{-1}(U) \rightarrow \mathbf{S}^{1},(s, h) \mapsto h\left(f_{s}\right)$.

Then, $\tau$ is a proper surjective map with fibers $\tau^{-1}(s)=\left(\mathbf{S}^{1}\right)^{r(s)}$, where $r(s):=$ $\operatorname{rank}\left(M^{\mathrm{gp}} / \mathcal{O}_{S}^{\times}\right)_{s}$. Note that $r(s)$ varies with $s \in S$.

We define the sheaf of logarithms $\mathcal{L}$ of $M^{\text {gp }}$ on $S^{\text {log }}$ as the fiber product:

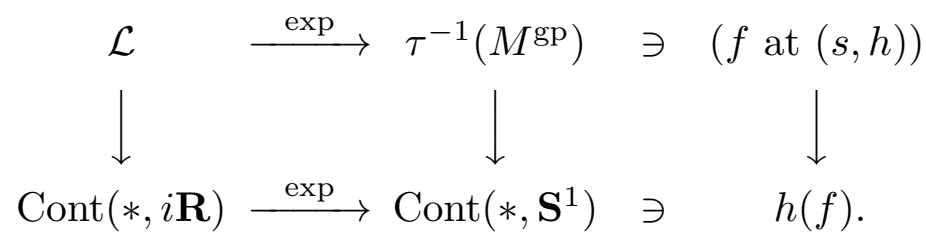

The commutative diagram

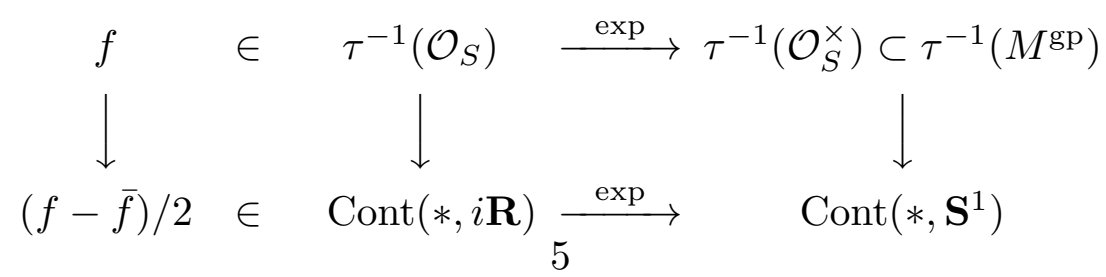


induces a homomorphism $\iota: \tau^{-1}\left(\mathcal{O}_{S}\right) \rightarrow \mathcal{L}$. We define

$$
\mathcal{O}_{S}^{\log }:=\frac{\tau^{-1}\left(\mathcal{O}_{S}\right) \otimes \operatorname{Sym}_{\mathbf{Z}}(\mathcal{L})}{\left(f \otimes 1-1 \otimes \iota(f) \mid f \in \tau^{-1}\left(\mathcal{O}_{S}\right)\right)} .
$$

Then, we have a morphism $\tau:\left(S^{\log }, \mathcal{O}_{S}^{\log }\right) \rightarrow\left(S, \mathcal{O}_{S}\right)$ of ringed spaces over $\mathbf{C}$.

For $s \in S$ and $t \in \tau^{-1}(s) \subset S^{\text {log }}$, let $t_{j}(1 \leq j \leq r(s))$ be elements of the stalk $\mathcal{L}_{t}$ such that the images in $\left(M^{\mathrm{gp}} / \mathcal{O}_{S}^{\times}\right)_{s}$ of $\exp \left(t_{j}\right)$ form a basis. Then $\mathcal{O}_{S, t}^{\text {log }}$ is a polynomial ring in $r(s)$ variables $t_{j}(1 \leq j \leq r(s))$ over $\mathcal{O}_{S, s}$ (cf. [KU09, Section 2.2.5]).

\subsection{Polarized log Hodge structure}

Let $S \in \mathcal{B}(\log )$.

A pre-polarized log Hodge structure (pre-PLH for short) of weight $w$ on $S$ is a triple $\left(H_{\mathbf{Z}},\langle\rangle,, H_{\mathcal{O}}\right)$ consisting of a locally constant sheaf of $\mathbf{Z}$-free modules of finite rank on $S^{\log }$, a nondegenerate $(-1)^{w}$-symmetric $\mathbf{Q}$-bilinear form on $H_{\mathbf{Q}}:=\mathbf{Q} \otimes H_{\mathbf{Z}}$, and a locally free $\mathcal{O}_{S}$-module on $S$ which satisfy the following conditions: There exists a specified isomorphism $\mathcal{O}_{S}^{\log } \otimes_{\mathbf{z}} H_{\mathbf{Z}} \simeq \mathcal{O}_{S}^{\log } \otimes_{\mathcal{O}_{S}} H_{\mathcal{O}}$, and there exists a specified decreasing filtration $F H_{\mathcal{O}}$ of $H_{\mathcal{O}}$ such that $F^{p} H_{\mathcal{O}}$ and $H_{\mathcal{O}} / F^{p} H_{\mathcal{O}}$ are locally free for all $p$. For $F^{p}:=\mathcal{O}_{S}^{\log } \otimes_{\mathcal{O}_{S}} F^{p} H_{\mathcal{O}}$, the orthoginality condition $\left.\left\langle F^{p}, F^{q}\right\rangle=0(p+q\rangle w\right)$ holds.

(Then, we have $\tau_{*} F^{p}=F^{p} H_{\mathcal{O}}$. Note that here we use the notation $\left(H_{\mathbf{Z}},\langle\rangle,, H_{\mathcal{O}}\right)$ instead of $\left(H_{\mathbf{Z}},\langle\rangle, F,\right)$ in [KU09, Definition 2.4.3].)

A pre-PLH on $S$ is a polarized log Hodge structure (PLH for short) on $S$ if its pullback to each $s \in S$ is a PLH on $s$ in the following sense. Let $\left(H_{\mathbf{Z}},\langle\rangle,, H_{\mathcal{O}}\right)$ be a pre-PLH on a fs log point $s$.

(1) $\nabla F^{p} \subset \omega_{s}^{1, \log } \otimes F^{p-1}$, where $\omega_{s}^{1, \log }$ is the sheaf of $\log$ differential 1-forms on $\left(s^{\log }, \mathcal{O}_{s}^{\log }\right)$ and $\nabla$ is the $\log$ Gauss-Manin connection defined by $\nabla:=d \otimes 1_{H_{\mathbf{Z}}}$ : $\mathcal{O}_{s}^{\log } \otimes H_{\mathbf{Z}} \rightarrow \omega_{s}^{1, \log \otimes H_{\mathbf{Z}}}$

(2) Let $t \in s^{\log }$, and $a: \mathcal{O}_{s, t}^{\log } \rightarrow \mathbf{C}$ be a specialization (i.e., a $\mathbf{C}$-algebra homomorphism). Put $F(a):=\mathbf{C} \otimes_{\mathcal{O}_{s, t}^{\log }} F_{t}$ which is a filtration on $H_{\mathbf{C}, t}$. Then $\left(H_{\mathbf{Z}, t},\langle,\rangle_{t}, F(a)\right)$ is a polarized Hodge structure in the usual sense if the specialization $a$ is sufficiently twisted in the following sense:

Fix a subset $\left(q_{j}\right)_{1 \leq j \leq n}$ of the log structure $M_{s}$ whose image in $M_{s} / \mathcal{O}_{s}^{\times}$generates $M_{s} / \mathcal{O}_{s}^{\times}$as monoid. A specialization $a$ is sufficiently twisted if $\exp \left(a\left(\log q_{j}\right)\right)$ is sufficiently near 0 for all $j$.

When $S$ is $\log$ smooth, a polarized log Hodge structure $\left(H_{\mathbf{z}},\langle\rangle,, H_{\mathcal{O}}\right)$ on $S$ is called a log variation of polarized Hodge structure if it satisfies the big Griffiths transversality condition:

$$
\left(d \otimes 1_{H_{\mathbf{C}}}\right) F^{p} \subset \omega_{S}^{1, \log } \otimes F^{p-1} \quad \text { for all } p,
$$

where $d \otimes 1_{H_{\mathbf{C}}}: \mathcal{O}_{S}^{\log } \otimes_{\mathbf{C}} H_{\mathbf{C}} \rightarrow \omega_{S}^{1, \log } \otimes_{\mathbf{C}} H_{\mathbf{C}}$ (cf. [KU09, Section 2.4.9]).

Let $S$ be as above. The condition (3) is stronger than the condition (1) imposed at every $\log$ point $s \in S$ endowed with the induced $\log$ structure $\left.M_{S}\right|_{s}$. When we need a contrast, we call the latter the small Griffiths transversality. 


\subsection{Nilpotent orbit and period map}

Let $D$ be the classifying space of polarized Hodge structures for a fixed data $\Lambda:=$ $\left(H_{0},\langle,\rangle_{0},\left(h^{p, q}\right)_{p+q=w}\right)$, and let $\check{D}$ be the "compact dual" ([G68]). For $A=\mathbf{Z}, \mathbf{Q}, \mathbf{R}, \mathbf{C}$, let $G_{A}:=\operatorname{Aut}\left(H_{0, A},\langle\rangle,\right)$ and $\mathfrak{g}_{A}:=\operatorname{End}\left(H_{0, A},\langle\rangle,\right)$.

Let $\sigma$ be a nilpotent cone in $\mathfrak{g}_{\mathbf{R}}$, i.e., a cone in $\mathfrak{g}_{\mathbf{R}}$ not containing a line and generated over $\mathbf{R}_{>0}$ by a finite number of mutually commutative nilpotent elements of $\mathfrak{g}_{\mathbf{R}}$. A subset $Z \subset \check{D}$ is a $\sigma$-nilpotent orbit if the following three conditions are satisfied for $F \in Z$.

(1) $Z=\exp \left(\sigma_{\mathbf{C}}\right) F$.

(2) $N F^{p} \subset F^{p-1}$ for any $N \in \sigma$ any $p$.

(3) If $N, \ldots, N_{n}$ generate $\sigma$, then $\exp \left(\sum_{j} i y_{j} N_{j}\right) F \in D$ for sufficiently large $y_{j}(\forall j)$.

Following [KU09, Ch. 2], we will briefly explain that the notion of a polarized log Hodge structure on an fs log point is equivalent to the notion of a nilpotent orbit. The basic idea is that, on the ringed space $\left(S^{\log }, \mathcal{O}_{S}^{\log }\right)$, any local system with unipotent monodromy becomes isomorphic to the constant sheaf of its fiber after shrinking $S$ and tensoring with $\mathcal{O}_{S}^{\log }$ (Proposition 2.3.2 loc.cit. and a list of errata loc.cit. on a web page of press.princeton.edu.).

Let $S \in \mathcal{B}(\log )$. Let $H=\left(H_{\mathbf{Z}},\langle\rangle,, H_{\mathcal{O}}\right)$ be a pre-polarized log Hodge structure on $S$ of Hodge type $\left(h^{p, q}\right)$ endowed with a $\Gamma$-level structure $\mu$, i.e., a section $\mu$ of the sheaf $\Gamma \backslash \mathcal{I} \operatorname{som}\left(\left(H_{\mathbf{Z}},\langle\rangle,\right),\left(H_{0},\langle,\rangle_{0}\right)\right)$ on $S^{\log }$. We recall from Section 2.5.3 loc.cit. the set-theoretic period map associated to $H$.

Let

$$
\check{D}_{\text {orb }}:=\left\{(\sigma, Z) \mid \sigma \text { is a nilpotent cone in } \mathfrak{g}_{\mathbf{R}}, Z=\exp \left(\sigma_{\mathbf{C}}\right) F \text { for some } F \in Z\right\} .
$$

Define a map

$$
\check{\varphi}: S \rightarrow \Gamma \backslash \check{D}_{\text {orb }}
$$

as follows. Let $s \in S$, and $t \in S^{\text {log }}$ lying over $s$, and let $\tilde{\mu}_{t}:\left(H_{\mathbf{Z}, t},\langle,\rangle_{t}\right) \stackrel{\sim}{\longrightarrow}\left(H_{0},\langle,\rangle_{0}\right)$ be a lifting of the germ $\mu_{t}$ of $\mu$ at $t$. Then, via $\tilde{\mu}_{t}$, the action of $\pi_{1}\left(s^{\log }\right)$ on $H_{\mathbf{Z}, t}$ defines a unilpotent action on the reference $\mathbf{Z}$-module $H_{0}$ preserving $\langle,\rangle_{0}$. Let $\sigma$ be the cone in $\mathfrak{g}_{\mathbf{R}}$ generated by the logarithms of the actions of elements of $\pi_{1}^{+}\left(s^{\log }\right)$ on $H_{0}$. Take generators $N_{j}(1 \leq j \leq n)$ of the cone $\sigma$, and let $h_{j} \in \operatorname{Hom}\left(M_{s} / \mathcal{O}_{s}^{\times}, \mathbf{N}\right) \simeq \pi_{1}^{+}\left(s^{\log }\right)$ be the element corresponding to $\exp N_{j}$. Take elements $q_{k} \in M_{s} \backslash \mathcal{O}_{s}^{\times}(1 \leq k \leq m)$ such that their images in $M_{s} / \mathcal{O}_{s}^{\times}$generate this monoid. Let $a_{0}, a \in \operatorname{sp}(t)$, and take $z_{j} \in \mathbf{C}$ $(1 \leq j \leq n)$ such that

$$
\frac{1}{2 \pi i}\left(a\left(\log q_{k}\right)-a_{0}\left(\log q_{k}\right)\right)=\sum_{j=1}^{n} z_{j} h_{j}\left(q_{k}\right) \quad(1 \leq k \leq m) .
$$

Then, by Proposition 2.5.1 loc.cit., we have

$$
F(a)=\exp \left(\sum_{j=1}^{n} z_{j} N_{j}\right) F\left(a_{0}\right)
$$


Hence $Z:=\left\{\tilde{\mu}_{t}(F(a)) \mid a \in \operatorname{sp}(t)\right\}=\exp \left(\sigma_{\mathbf{C}}\right) F\left(a_{0}\right)$ in $\check{D}$. We define

$$
\check{\varphi}(s):=((\sigma, Z) \bmod \Gamma) \in \Gamma \backslash \check{D}_{\text {orb }}
$$

The point $\check{\varphi}(s)$ is independent of the choices of $t$ and $\tilde{\mu}_{t}$. If $S^{\prime} \rightarrow S$ in $\mathcal{B}(\log )$ is strict (i.e., the $\log$ structure of $S^{\prime}$ coincides with the pullback of that of $S$ ), then by using the pullback of $H$ to $S^{\prime}$ we have a commutative diagram

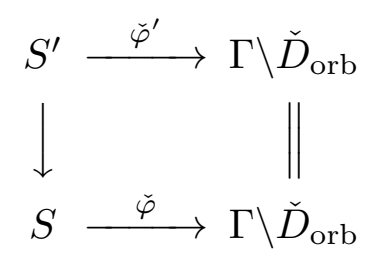

(Lemma 2.5.4 loc.cit.).

Positivity conditions 1.3 (2) and 1.4 (3) coincide (Proposition 2.5.5 (ii) loc.cit.).

Griffiths transversality conditions 1.3 (1) and 1.4 (2) coincide, because the composition $H^{0}\left(s^{\log }, \mathcal{O}_{s}^{\log } \otimes H_{\mathbf{Z}}\right) \rightarrow \mathcal{O}_{s}^{\log } \otimes H_{\mathbf{Z}} \stackrel{a}{\rightarrow} H_{\mathbf{C}, t}$ is an isomorphism (Proposition 2.5.5 (i) loc.cit.).

\subsection{Toric variety}

Let $\sigma$ be a nilpotent cone in $\mathfrak{g}_{\mathbf{R}}$ from 1.4. Assume that the cone generators of $\sigma$ can be taken from $\mathfrak{g}_{\mathbf{Q}}$. Let $\Gamma$ be a subgroup of $G_{\mathbf{Z}}$. Define a monoid $\Gamma(\sigma):=\Gamma \cap \exp (\sigma)$ and the dual monoid $P(\sigma):=\Gamma(\sigma)^{\vee}=\operatorname{Hom}(\Gamma(\sigma), \mathbf{N})$. Define a toric variety and a torus by

$$
\operatorname{toric}_{\sigma}:=\operatorname{Hom}\left(P(\sigma), \mathbf{C}^{\text {mult }}\right) \supset \operatorname{torus}_{\sigma}:=\operatorname{Hom}\left(P(\sigma)^{\mathrm{gp}}, \mathbf{C}^{\times}\right),
$$

where $\mathbf{C}^{\text {mult }}$ is $\mathbf{C}$ regarded as a monoid with multiplication and $P(\sigma)^{\text {gp }}$ is the group generated by the monoid $P(\sigma)$. The exponential sequence $0 \rightarrow \mathbf{Z} \rightarrow \mathbf{C} \rightarrow \mathbf{C}^{\times} \rightarrow 1$ induces the universal covering of the torus

$$
0 \rightarrow \operatorname{Hom}\left(P(\sigma)^{\mathrm{gp}}, \mathbf{Z}\right) \rightarrow \operatorname{Hom}\left(P(\sigma)^{\mathrm{gp}}, \mathbf{C}\right) \stackrel{\text { e }}{\rightarrow} \operatorname{Hom}\left(P(\sigma)^{\mathrm{gp}}, \mathbf{C}^{\times}\right) \rightarrow 1,
$$

where $\operatorname{Hom}\left(P(\sigma)^{\mathrm{gp}}, \mathbf{Z}\right)=\Gamma(\sigma)^{\mathrm{gp}}$ is considered as the fundamental group of torus $\sigma$, and $\mathbf{e}(z \otimes \gamma):=e^{2 \pi i z} \otimes \gamma\left(z \in \mathbf{C}, \gamma \in \Gamma(\sigma)^{\mathrm{gp}}=\operatorname{Hom}\left(P(\sigma)^{\mathrm{gp}}, \mathbf{Z}\right)\right)$.

Let $\rho$ be a face of the cone $\sigma$ in the usual sense. Then, we have a surjective homomorphism $P(\rho) \leftarrow P(\sigma)$ and hence an closed immersion toric im $\hookrightarrow$ toric $_{\sigma}$. The origin $0_{\rho} \in$ toric $_{\rho}$ is the monoid homomorphism sending 1 to 1 and all the other elements of $P(\rho)$ to 0 , which is viewed as a point of toric $\sigma$ by the above immersion. Then, as a set, we have

$$
\text { toric }_{\sigma}=\left\{\mathbf{e}(z) 0_{\rho} \mid \rho \prec \sigma, z \in \sigma_{\mathbf{C}} /\left(\rho_{\mathbf{C}}+\log \Gamma(\sigma)^{\mathrm{gp}}\right)\right\} .
$$

Here $\mathbf{e}(c \log \gamma):=\mathbf{e}(c \otimes \gamma)=e^{2 \pi i} \otimes \gamma\left(c \in \mathbf{C}, \gamma \in \Gamma(\sigma)^{g p}\right)$. 
For $S:=$ toric $_{\sigma}$, polar coordinate $\mathbf{R}_{\geq 0} \times \mathbf{S}^{1} \rightarrow \mathbf{R}_{\geq 0} \mathbf{S}^{1}=\mathbf{C}$ induces $\tau: S^{\log } \rightarrow S$ as

$$
\begin{aligned}
\tau: S^{\log } & =\operatorname{Hom}\left(P(\sigma), \mathbf{R}_{\geq 0}^{\text {mult }}\right) \times \operatorname{Hom}\left(P(\sigma), \mathbf{S}^{1}\right) \\
& =\left\{\left(\mathbf{e}(i y) 0_{\rho}, \mathbf{e}(x)\right) \mid \rho \prec \sigma, x \in \sigma_{\mathbf{R}} /\left(\rho_{\mathbf{R}}+\log \Gamma(\sigma)^{\mathrm{gp}}\right), y \in \sigma_{\mathbf{R}} / \rho_{\mathbf{R}}\right\} \\
& \rightarrow S=\operatorname{Hom}\left(P(\sigma), \mathbf{C}^{\text {mult }}\right), \quad \tau\left(\mathbf{e}(i b) 0_{\rho}, \mathbf{e}(a)\right)=\mathbf{e}(a+i b) 0_{\rho} .
\end{aligned}
$$

Since $0 \rightarrow \rho_{\mathbf{R}} / \log \Gamma(\rho)^{\mathrm{gp}} \rightarrow \sigma_{\mathbf{R}} / \log \Gamma(\sigma)^{\mathrm{gp}} \rightarrow \sigma_{\mathbf{R}} /\left(\rho_{\mathbf{R}}+\log \Gamma(\sigma)^{\mathrm{gp}}\right) \rightarrow 0$ is exact, the fiber of $\tau$, as a set, is described as

$$
\tau^{-1}\left(\mathbf{e}(a+i b) 0_{\rho}\right)=\left\{\left(\mathbf{e}(i b) 0_{\rho}, \mathbf{e}(a+x)\right) \mid x \in \rho_{\mathbf{R}} / \log \Gamma(\rho)^{\mathrm{gp}}\right\} \simeq\left(\mathbf{S}^{1}\right)^{r},
$$

where $r=r(\rho):=\operatorname{rank} \rho$ varies with $\rho \prec \sigma$.

\subsection{Moduli of polarized log Hodge structures of specified type}

Let $\Phi=\left(\Lambda, \sigma, \Gamma(\sigma)^{\mathrm{gp}}\right)$, where $\Lambda$ is from $1.4, \sigma$ is a nilpotent cone from 1.5, and $\Gamma$ is a subgroup of $G_{\mathbf{Z}}$.

Let $S:=\operatorname{toric}_{\sigma}=\operatorname{Hom}(\Gamma(\sigma), \mathbf{C})=\operatorname{Spec}(\mathbf{C}[P(\sigma)])_{\text {an }}(1.5)$. Let $H_{\sigma}=\left(H_{\sigma, \mathbf{z}},\langle\rangle,\right)$ be the canonical locally constant sheaf on $S^{\log }$ given by the representation of $\pi_{1}\left(S^{\log }\right)=$ $\Gamma(\sigma)^{\mathrm{gp}} \subset G_{\mathbf{Z}}$ on $\left(H_{0},\langle,\rangle_{0}\right)$. The universal pre-PLH $H$ on $\check{E}_{\sigma}:=$ toric $_{\sigma} \times \check{D}$ is given by $H_{\sigma}$ together with the isomorphism $\mathcal{O}_{\check{E}_{\sigma}}^{\log } \otimes_{\mathbf{Z}} H_{\sigma}=\mathcal{O}_{\check{E}_{\sigma}}^{\log } \otimes_{\mathcal{O}_{\check{E}_{\sigma}}} H_{\mathcal{O}}$, where $H_{\mathcal{O}}:=\mathcal{O}_{\check{E}_{\sigma}} \otimes H_{0}$ is the free $\mathcal{O}_{\check{E}_{\sigma}}$-module coming from that on $\check{D}$ endowed with the universal Hodge filtration $F$. Define

$$
E_{\sigma}:=\left\{x \in \check{E}_{\sigma} \mid H(x) \text { is a PLH }\right\} .
$$

Note that slits appear in $E_{\sigma}$ because of the small Griffiths transversality 1.3 (1), or equivalently 1.4 (2), and Positivity 1.3 (2), or equivalently 1.4 (3).

As a set, we define

$$
D_{\sigma}:=\left\{(\rho, Z) \in \check{D}_{\text {orb }} \mid(\rho, Z) \text { is a nilpotent orbit with } \rho \in \text { face }(\sigma)\right\}
$$

A structure as an object of $\mathcal{B}(\log )$ on $\Gamma(\sigma)^{\mathrm{gp}} \backslash D_{\sigma}$ is introduced by the diagram:

$$
\begin{gathered}
E_{\sigma} \stackrel{\text { PLH }}{\subset} \quad \check{E}=\text { toric }_{\sigma} \times \check{D} \\
\qquad \sigma_{\mathrm{C}_{\text {-torsor }}} \\
\Gamma(\sigma)^{\mathrm{gp} \backslash D_{\sigma}}
\end{gathered}
$$

Here the action of $h \in \sigma_{\mathbf{C}}$ on $\left(\mathbf{e}(a) 0_{\rho}, F\right) \in E_{\sigma}$ is $\left(\mathbf{e}(h+a) 0_{\rho}, \exp (-h) F\right)$, and the projection to the quotient is described as $\left(\mathbf{e}(a) 0_{\rho}, F\right) \mapsto\left(\rho, \exp \left(\rho_{\mathbf{C}}+a\right) F\right) \bmod \Gamma(\sigma)^{\mathrm{gp}}$. By using the Fundamental Diagram from Introduction, we transported good properties of the Borel-Serre type compactification $\Gamma \backslash D_{\mathrm{BS}}$, such as Hausdorffness, to the $\sigma_{\mathbf{C}}$-action on $E_{\sigma}$ and showed that the projection $E_{\sigma} \rightarrow E_{\sigma} / \sigma_{\mathbf{C}}=\Gamma(\sigma)^{g p} \backslash D_{\sigma}$ is a $\sigma_{\mathbf{C}}$-torsor in the category $\mathcal{B}(\log )$. This is the hardest part of the proof in [KU09]. 
Let $S \in \mathcal{B}(\log )$. A polarized log Hodge structure of type $\Phi$ on $S$ is a pre-PLH $H=$ $\left(H_{\mathbf{Z}},\langle\rangle,, H_{\mathcal{O}}\right)$ endowed with a $\Gamma$-level structure $\mu$ satisfying the following condition: For any $s \in S$, any $t \in \tau^{-1}(s)=s^{\log }$, and any representative $\tilde{\mu}_{t}: H_{\mathbf{Z}, t} \stackrel{\sim}{\rightarrow} H_{0}$, there exists $\rho \in$ face $(\sigma)$ such that $\rho$ contains $\tilde{\mu}_{t} P_{s} \tilde{\mu}_{t}^{-1}$ and that $\left(\rho, \tilde{\mu}_{t}\left(\mathbf{C} \otimes_{\mathcal{O}_{S, t}^{\log }} F_{t}\right)\right)$ generates a nilpotent orbit, where $P_{s}:=\operatorname{Image}\left(\operatorname{Hom}\left(\left(M_{S} / \mathcal{O}_{S}^{\times}\right)_{s}, \mathbf{N}\right) \hookrightarrow \pi_{1}\left(s^{\log }\right) \rightarrow \operatorname{Aut}\left(H_{\mathbf{Z}, t}\right)\right)$ is the local monodromy monoid of $H_{\mathbf{Z}}$ at $s$. (Then, the smallest such face $\rho$ exists.)

As explained in 1.4, a polarized log Hodge structure of type $\Phi$ is a polarized log Hodge structure.

Theorem. (i) $\Gamma(\sigma)^{\mathrm{gp}} \backslash D_{\sigma} \in \mathcal{B}(\log )$, which is a Hausdorff log manifold.

(ii) On $\mathcal{B}(\log ), \Gamma(\sigma)^{\mathrm{gp}} \backslash D_{\sigma}$ represents the functor $\underline{\mathrm{PLH}}_{\Phi}$ of PLH of type $\Phi$.

Log period map. Let $S \in \mathcal{B}(\log )$. Then we have an isomorphism

$$
\begin{aligned}
& \underline{\mathrm{PLH}}_{\Phi}(S) \stackrel{\sim}{\rightarrow} \operatorname{Map}\left(S, \Gamma(\sigma)^{\mathrm{gp}} \backslash D_{\sigma}\right), \\
& H \mapsto\left(S \ni s \mapsto\left(\rho, \exp \left(\rho_{\mathbf{C}}\right) \tilde{\mu}_{t}\left(\mathbf{C} \otimes_{\mathcal{O}_{S, t}^{\log }} F_{t}\right)\right) \bmod \Gamma(\sigma)^{\mathrm{gp}}\right)
\end{aligned}
$$

which is functorial in $S$.

When $S$ is $\log$ smooth and $H$ is a log variation of polarized Hodge structure (1.3), the associated period map $S \rightarrow \Gamma(\sigma)^{\mathrm{gp}} \backslash D_{\sigma}$ is called a horizontal log period map.

\section{Calabi-Yau threefolds}

Let $V$ be a Calabi-Yau threefold and $V^{\circ}$ be its mirror Calabi-Yau threefold.

We recall some results for A-model of $V$ and B-model of $V^{\circ}$ mainly using the notation from [CK99]. We introduce $\mathbf{Z}$-structure on the ringed space $\left(S^{\log }, \mathcal{O}_{S}^{\log }\right)$, and prove the equivalence of the formulation (4) with the other formulations (1)-(3) in Introduction.

\subsection{Picard-Fuchs ideal}

We review here [CK99, Section 5.1.2]. Let $f: X \rightarrow S^{*}$ be a proper smooth family of Kähler manifolds of dimension 3 over a smooth base $S^{*}$, and let $H$ be the variation of Hodge structure over $S^{*}$ arising from this family on the third relative cohomology sheaf $R^{3} f_{*} \mathbf{Z}$. Let $\mathcal{D}$ be the sheaf of rings of holomorphic differential operators on $S^{*}$. The Gauss-Manin connection $\nabla$ on $H$ gives the $\mathcal{D}$-module structure on $H$. Let $\Omega$ be a fixed local section of the Hodge filter $F^{3}$ at a point $p \in S^{*}$. Let $z_{1}, \ldots, z_{r}$ be local coordinates of $S^{*}$ at $p$. Then, locally on $S^{*}, \mathcal{D}=\mathbf{C}\left\{z_{1}, \ldots, z_{r}\right\}\left[\partial / \partial z_{1}, \ldots, \partial / \partial z_{r}\right]$ and the Gauss-Manin connection $\nabla$ defines an $\mathcal{O}_{S^{*}}$-homomorphism

$$
\phi: \mathcal{D} \rightarrow F^{0} \quad \text { by } \quad \phi\left(X_{1} \cdots X_{m}\right):=\nabla_{X_{1}} \cdots \nabla_{X_{m}} \Omega
$$

for vector fields $X_{i}$ on $S^{*}$. The ideal $I:=\operatorname{Ker}(\phi)$ is called the Picard-Fuchs ideal. Since

$$
\frac{\partial}{\partial z_{j}} \int_{\gamma} \Omega=\int_{\gamma} \nabla_{\partial / \partial z_{j}} \Omega
$$


for a locally constant homology 3-cycle $\gamma$, the periods $y:=\int_{\gamma} \Omega$ of $\Omega$ are solutions of the equations $D y=0$ for all $D \in I$.

By analytic continuation, the above things make sense as algebraic differential equations over all $S^{*}$ if we restrict our attention to the periods of algebraic differentials.

\subsection{B-model of $V^{\circ}$}

We recall the definition of maximally unipotent monodromy point from [M93], cf. [CK99, Section 5.2].

Let $S^{*}:=\mathcal{M}\left(V^{\circ}\right)$ be the full complex moduli space of $V^{\circ}$. Then $S^{*}$ is a smooth manifold of dimension $r:=h^{2,1}\left(V^{\circ}\right)$. Assume that $S^{*}$ has a smooth compactification $S:=\overline{\mathcal{M}}\left(V^{\circ}\right)$ with normal crossing boundary divisor $D=D_{1}+\cdots+D_{r}:=S \backslash S^{*}$ with $D_{j}=\left\{z_{j}=0\right\}(1 \leq j \leq r)$. The point $p_{0}:=D_{1} \cap \cdots \cap D_{r}=\left(z_{1}=\cdots=z_{r}=0\right)$ is a maximally unipotent monodromy point satisfying Integrality Conjecture if the following conditions hold.

(1) The monodromy transformations $\mathcal{T}_{j}$ are all unipotent.

(2) Put $N_{j}:=\log \mathcal{T}_{j}$ and $N=\sum_{j} a_{j} N_{j}$ with all $a_{j}>0$. If $W=W(N)$ is the monodromy weight filtration, then $\operatorname{dim} W_{0}=\operatorname{dim} W_{1}=1$ and $\operatorname{dim} W_{2}=1+r$.

(3) We use the $\mathbf{Z}$-structure $W_{k, \mathbf{Z}}$ of the locally constant sheaf $W_{k}$, which will be defined in 2.4 below. Let $g_{0}, \ldots, g_{r}$ be a local flat $\mathbf{Z}$-basis of $W_{2, \mathbf{Z}}$ such that $g_{0}$ spans $W_{0, \mathbf{Z}}$ and define $m_{j k}$ via $N_{j} g_{k}=m_{j k} g_{0}$ for $1 \leq j, k \leq r$. Then the matrix $\left(m_{j k}\right)$ has the inverse $\left(m^{j k}\right)$ over $\mathbf{Z}$ (This is Intergrality Conjecture in [M97]).

Let $g_{0}, \ldots, g_{r}$ and $m^{j k}$ be as in (3), and let $\Omega$ be a fixed local section of the Hodge filter $F^{3}$ with log pole over the boundary. Let $\gamma_{j}$ be the homology class corresponding to $g_{j}$ by the Poincaré duality $(0 \leq j \leq r)$. Then,

$$
y_{0}:=\left\langle g_{0}, \Omega\right\rangle=\int_{\gamma_{0}} \Omega, \quad y_{k}:=\sum_{j=1}^{r}\left\langle g_{j}, \Omega\right\rangle m^{j k}=\sum_{j=1}^{r}\left(\int_{\gamma_{j}} \Omega\right) m^{j k} \quad(1 \leq k \leq r)
$$

are the first half of a basis of the solutions of the Picard-Fuchs equation. Let $t_{k}:=y_{k} / y_{0}$ and $u_{k}:=t_{k} / 2 \pi i(1 \leq k \leq r)$, which are called canonical parameters. The canonical coordinates at $p_{0}$ are given by $q_{k}:=\exp \left(t_{k}\right)=\exp \left(2 \pi i u_{k}\right)(1 \leq k \leq r)$ which yield the distinguished chart of the $\log$ structure on $S$ induced by the divisor $D$ and give the mirror map. (For the uniqueness, see [CK99, Section 6.3, pp.150-151].)

The relation with the coordinates $z_{k}$ is as follows. Define $\tilde{y}_{k}$ by

$$
y_{k}=y_{0} \frac{\log z_{k}}{2 \pi i}+\tilde{y}_{k},
$$

then $\tilde{y}_{k}$ is holomorphic at $p_{0}$ with $\tilde{y}_{k}(p)=0$ and

$$
q_{k}=\exp \left(2 \pi i y_{k} / y_{0}\right)=z_{k} \exp \left(2 \pi i \tilde{y}_{k} / y_{0}\right) .
$$

Let $\delta_{j}:=\partial / \partial u_{j}(1 \leq j \leq r)$. Then, the normalized Yukawa couplings $Y_{j k l}$ are expressed as

$$
Y_{j k l}=-\int_{V^{\circ}} \tilde{\Omega} \wedge \nabla_{\delta_{j}} \nabla_{\delta_{k}} \nabla_{\delta_{l}} \tilde{\Omega} \quad(1 \leq j, k, l \leq r),
$$


where $\tilde{\Omega}:=\Omega / y_{0}$ is the normalized holomorphic 3 -form, and $\nabla=\tau_{*}\left(d \otimes 1_{H_{\mathbf{Z}}}\right)$ is the $\log$ Gauss-Manin connection. There exists a holomorphic function $\Phi=\Phi_{V^{\mathrm{o}}}^{\mathrm{GM}}$ in $u_{1}, \ldots, u_{r}$ uniquely determined up to quardratic terms by the property

$$
Y_{j k l}=\frac{1}{(2 \pi i)^{3}} \frac{\partial^{3} \Phi}{\partial u_{j} \partial u_{k} \partial u_{l}}
$$

Such $\Phi=\Phi_{V^{\circ}}^{\mathrm{GM}}$ is called a Gauss-Manin potential of $V^{\circ}$. These are written in [CK99, Sect. 5.6.3]. There are some unclear points in the proof of Proposition 5.6.1 and the proceeding explanation for the case of higher dimensional moduli loc. cit., but we can rescue them by [KU09, Prop. 2.5.5] and the $\mathbf{Z}$-structure introduced in Section 2.4 below (cf. 2.5).

\subsection{A-model of $V$}

We recall the following notion from [CK99, Section 8.5]. Let $T_{1}, \ldots, T_{r}$ be an integral basis in the closure of the Kähler cone $K(V)$ of $V$, and let $\sigma:=\sum_{j=1}^{r} \mathbf{R}_{\geq 0} T_{j}$ be the simplicial cone generated by them. Then $\operatorname{Int}(\sigma) \subset K(V)$. Let $u_{1}, \ldots, u_{r}$ be the coordinates of $K_{\mathbf{C}}(V)=\bigoplus_{j=1}^{r} \mathbf{C} T_{j}$, and let

$$
\mathcal{D}_{\sigma}:=\left(H^{2}(V, \mathbf{R})+i \operatorname{Int}(\sigma)\right) / \operatorname{Image} H^{2}(V, \mathbf{Z}) .
$$

Then,

$$
u_{1} T_{1}+\cdots+u_{r} T_{r} \mapsto\left(q_{1}, \ldots, q_{r}\right)=\left(e^{2 \pi i u_{1}}, \ldots, e^{2 \pi i u_{r}}\right)
$$

induces a biholomorphic map $\mathcal{D}_{\sigma} \stackrel{\sim}{\longrightarrow}\left(\Delta^{*}\right)^{r}$, and via the inclusion $\left(\Delta^{*}\right)^{r} \subset \Delta^{r}$, we can regard $q_{0}:=0 \in \Delta^{r}$ as a large radius limit point in the terminology of [loc.cit., Ch. 6]. We assume $\mathcal{K} \mathcal{M}(V)=\mathcal{D}_{\sigma}=\left(\Delta^{*}\right)^{r}$ and $\overline{\mathcal{K} \mathcal{M}}(V)=\Delta^{r}$.

For $\beta \in H_{2}(V, \mathbf{Z})$, denote $q^{\beta}:=q^{\int_{\beta} T_{1}} \cdots q^{\int_{\beta} T_{r}}$. Then, the Gromov-Witten potential of $V$ is defined by

$$
\Phi_{V}^{\mathrm{GW}}:=\frac{1}{6} \int_{V}\left(\sum_{j=1}^{r} t_{j} T_{j}\right)^{3}+\sum_{0 \neq \beta \in H_{2}(V, \mathbf{Z})}\left\langle I_{0,0, \beta}\right\rangle q^{\beta}=\frac{(2 \pi i)^{3}}{6} \int_{V}\left(\sum_{j=1}^{r} u_{j} T_{j}\right)^{3}+\left(\Phi_{\mathrm{GW}}^{V}\right)_{\mathrm{hol}}
$$

([loc.cit., Formula (8.51)]). Here $t_{j}:=2 \pi i u_{j}$, and $N_{\beta}:=\left\langle I_{0,0, \beta}\right\rangle$ is the Gomov-Witten invariant whose definition see, e.g. [loc.cit., Ch. 7]. As in the proof of [loc.cit., Proposition 8.5.3], we can restrict the sum over $\beta \neq 0$ which are effective and satisfy $\int_{\beta} T_{j}>0$ for all $j$. As in [loc.cit., Section 8.5.3], we assume that $\left(\Phi_{V}^{\mathrm{GW}}\right)_{\text {hol }}$ is a convergent power series in the $q_{j}$ near $0 \in \Delta^{r}$. Then $\left(\Phi_{V}^{\mathrm{GW}}\right)_{\text {hol }}$ is a holomorphic function which vanishes at $0 \in \Delta^{r}$.

There exist unique rational numbers $n_{\beta}$, called instanton numbers, such that

$$
N_{\beta}=\sum_{\beta=k \gamma} n_{\gamma} k^{-3}
$$


where $k$ runs through positive integer and $\gamma \in H_{2}(V, \mathbf{Z})$. Then, the three points correlation function $\left\langle\alpha_{1}, \alpha_{2}, \alpha_{3}\right\rangle\left(\alpha_{i} \in H^{2}(V, \mathbf{C})\right)$ of A-model of $V$ is described as

$$
\begin{aligned}
\left\langle\alpha_{1}, \alpha_{2}, \alpha_{3}\right\rangle: & =\sum_{\beta \in H_{2}(V, \mathbf{Z})}\left\langle I_{0,3, \beta}\right\rangle\left(\alpha_{1}, \alpha_{2}, \alpha_{3}\right) q^{\beta} \\
& =\left\langle I_{0,3,0}\right\rangle\left(\alpha_{1}, \alpha_{2}, \alpha_{3}\right)+\sum_{0 \neq \beta \in H_{2}(V, \mathbf{Z})}\left\langle I_{0,0, \beta}\right\rangle q^{\beta} \int_{\beta} \alpha_{1} \int_{\beta} \alpha_{2} \int_{\beta} \alpha_{3} \\
& =\int_{V} \alpha_{1} \cup \alpha_{2} \cup \alpha_{3}+\sum_{0 \neq \beta \in H_{2}(V, \mathbf{Z})} n_{\beta} \frac{q^{\beta}}{1-q^{\beta}} \int_{\beta} \alpha_{1} \int_{\beta} \alpha_{2} \int_{\beta} \alpha_{3}
\end{aligned}
$$

(cf., e.g. [loc.cit., Section 7.4.4]). Hence, by using $\Phi=\Phi_{V}^{\mathrm{GM}}$, we have

$$
\left\langle T_{j}, T_{k}, T_{l}\right\rangle=\frac{\partial^{3} \Phi}{\partial t_{j} \partial t_{k} \partial t_{l}}=\frac{1}{(2 \pi i)^{3}} \frac{\partial^{3} \Phi}{\partial u_{j} \partial u_{k} \partial u_{l}}
$$

(cf., e.g. [loc.cit., Formula (8.34)]).

\subsection{Z-structure}

Let $S^{*}$ be $\mathcal{K} \mathcal{M}(V)$ for A-model of $V$ and $\mathcal{M}\left(V^{\circ}\right)$ for B-model for $V^{\circ}$, and let $S$ be $\overline{\mathcal{K} \mathcal{M}}(V)$ for A-model and $\overline{\mathcal{M}}\left(V^{\circ}\right)$ for B-model. Endow $S$ with the log structure associated to the divisor $S \backslash S^{*}$. Consider a diagram:

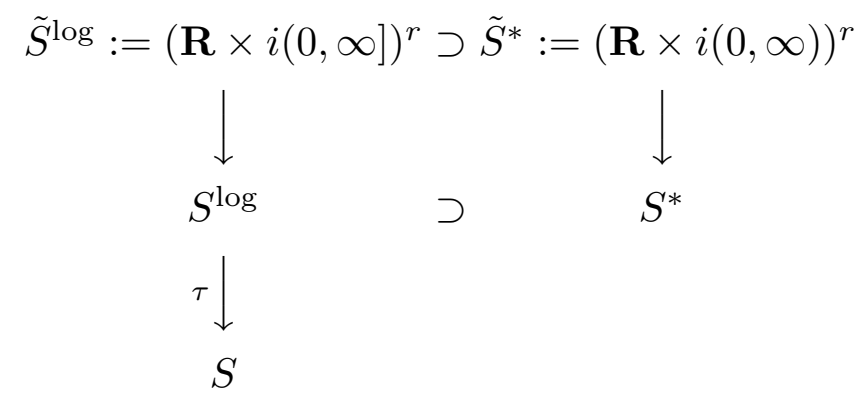

The coordinates $u=\left(u_{j}\right)_{1 \leq j \leq r}$ of $\tilde{S}^{*}$ extends over $\tilde{S}^{\log }$. Fix base points as $u_{0}=$ $\left(u_{0, j}\right)_{1 \leq j \leq r}:=(0+i \infty)^{r} \in \widetilde{S}^{\log } \mapsto b:=(\overline{0}+i \infty)^{r} \in S^{\log } \mapsto q=0=(0)^{r} \in S$, where $q=0$ corresponds to $q_{0}$ for A-model and $p_{0}$ for B-model. Note that to fix a base point $u=u_{0}$ on $\tilde{S}^{\log }$ is equivalent to fix a base point $b$ on $S^{\log }$ and also branches of $(2 \pi i)^{-1} \log q_{j}(1 \leq j \leq r)$.

We first consider B-model $\mathcal{H}^{V^{\circ}}$ of $V^{\circ}$. Let $f: X \rightarrow S^{*}$ be the smooth projective family of Calabi-Yau threefolds over a punctured neighborhood of the maximally unipotent monodromy point. Let $\mathcal{H}_{\mathbf{Z}}{ }^{\circ}=R^{3} f_{*} \mathbf{Z}$, and extend this to the local system of Z-modules over $S^{\log }$ (cf. [KU09, Proposition 2.3.5], [O03]), still denoted $\mathcal{H}_{\mathbf{Z}}^{V^{\circ}}$. Let $N_{1}, \ldots, N_{r}$ be the monodromy logarithm at the maximally unipotent monodromy point, and $W=W\left(\sum_{j=1}^{r} N_{j}\right)$ be the monodromy weight filtration. Define $W_{k, \mathbf{Z}}:=W_{k} \cap \mathcal{H}_{\mathbf{Z}}^{V^{\circ}}$ for all $k$. 
Log Hodge theoretic description of $\mathcal{H}_{\mathbf{Z}}^{V^{\circ}}$ is as follows. Let $\left(S^{\log }, \mathcal{O}_{S}^{\log }\right)$ be the ringed space in 1.2. Then, for a local section $s \in \mathcal{O}_{S}^{\log } \otimes_{\mathcal{O}} \mathcal{H}_{\mathcal{O}}^{V^{\circ}}$, the following statements are equivalent:

(1) $s$ belongs to $\mathcal{H}_{\mathbf{Z}}^{V^{\circ}}$.

(2) $\nabla s=0\left(\nabla=\nabla^{\mathrm{GM}}\right)$ and $s(b) \in \mathcal{H}_{\mathbf{Z}}^{V^{\circ}}(b)$ for the base point $b \in S^{\log }$.

Now we consider A-model $\mathcal{H}^{V}$ of $V$. Let $\nabla=\nabla^{\text {middle }}$ be the A-model connection from 2.5 (3A) below. For $s \in \mathcal{O}_{S}^{\log } \otimes_{\mathcal{O}} \mathcal{H}_{\mathcal{O}}^{V}$, define $s \in \mathcal{H}_{\mathbf{Z}}^{V}$ if $\nabla s=0$ and $s(b)$ belongs to the fiber over the base point $b \in S^{\log }$ of the extension of $\hat{\Gamma}$-integral structure of Iritani in $[4]$

For both models, let $B:=\mathcal{H}_{\mathbf{Z}}\left(u_{0}\right)=\mathcal{H}_{\mathbf{Z}}(b)$. Let $b_{0}, b_{1}, \ldots, b_{r},-b^{0}, b^{1}, \ldots, b^{r}$ be symplectic bases of $B$, for $S$ in A-model in 2.5 (1A) below and for $Q$ in B-model in 2.5 (1B) below.

\subsection{Correspondence table}

Specify the canonical parameters $u_{1}, \ldots, u_{r}$, or equivalently specify $t_{1} \ldots, t_{r}$, and let $\Phi_{V}^{\mathrm{GW}}$ be as in 2.3. In this section, we assume $\Phi_{V}^{\mathrm{GW}}=\Phi_{V^{\circ}}^{\mathrm{GM}}$, denoted by the same $\Phi$, and, expanding [CK99, Sections 8.5-8.6], we write down a correspondence table on the ringed space $\left(S^{\log }, \mathcal{O}_{S}^{\log }\right)$.

(1A) Polarization of $A$-model of $V$.

$$
S(\alpha, \beta):=(-1)^{p} \int_{V} \alpha \cup \beta \quad\left(\alpha \in H^{p, p}(V), \beta \in H^{3-p, 3-p}(V)\right) .
$$

(1B) Polarization of B-model of $V^{\circ}$.

$$
Q(\alpha, \beta):=(-1)^{3(3-1) / 2} \int_{V^{\circ}} \alpha \cup \beta=-\int_{V^{\circ}} \alpha \cup \beta \quad\left(\alpha, \beta \in H^{3}\left(V^{\circ}\right)\right) .
$$

(2A) Specified sections inducing $\mathbf{Z}$-basis of graded quotients of A-model of $V$.

We take

$$
\begin{aligned}
& T_{0}:=[V]=1 \in H^{0}(V, \mathbf{Z}), T_{i} \in H^{2}(V, \mathbf{Z})(1 \leq i \leq r), \\
& T^{i} \in H^{4}(V, \mathbf{Z})(1 \leq i \leq r), T^{0}:=[\mathrm{pt}] \in H^{6}(V, \mathbf{Z}),
\end{aligned}
$$

such that $S\left(T_{0}, T^{0}\right)=1$ and $S\left(T_{i}, T^{j}\right)=-\delta_{i, j}$. Hence, $T_{0}, T_{1}, \ldots, T_{r},-T^{0}, T^{1}, \ldots$, $T^{r}$ form a symplectic basis for $S$.

(2B) Specified sections inducing Z-basis of $\mathrm{gr}^{M}$ for B-model of $V^{\circ}$.

We use Deligne decomposition [2]. We consider $B=\mathcal{H}_{\mathbf{Z}, u_{0}}=\mathcal{H}_{\mathbf{Z}, b}$ in 3.4 as a constant sheaf on $S^{\log }$. We have locally free $\mathcal{O}_{S}$-submodules $\mathcal{W}_{2 p}:=\tau_{*}\left(\mathcal{O}_{S}^{\log } \otimes_{\mathbf{z}} W_{2 p} B\right)$ 
and $\mathcal{F}^{p}$ of $\tau_{*}\left(\mathcal{O}_{S}^{\log } \otimes_{\mathbf{z}} B\right)=\mathcal{O}_{S} \otimes_{\mathbf{z}} B$. The mixed Hodge structure of Hodge-Tate type $(\mathcal{W}, \mathcal{F})$ has decomposition:

$$
\mathcal{O}_{S} \otimes \mathbf{z} B=\bigoplus_{p} I^{p, p}, \quad I^{p, p}:=\mathcal{W}_{2 p} \cap \mathcal{F}^{p} \stackrel{\sim}{\rightarrow} \operatorname{gr}_{2 p}^{\mathcal{W}}
$$

Transporting the basis $b_{0}, b_{1}, \ldots, b_{r}, b^{1}, \ldots, b^{r}, b^{0}$ of $B$ via isomorphism

$$
I^{p, p} \stackrel{\sim}{\longrightarrow} \mathcal{O}_{S} \otimes_{\mathbf{Z}} \operatorname{gr}_{2 p}^{W} B
$$

we define sections

$$
e_{0}:=\tilde{\Omega} \in I^{3,3}, e_{i} \in I^{2,2}(1 \leq i \leq r), e^{i} \in I^{1,1}(1 \leq i \leq r), e^{0}=g_{0} \in I^{0,0} .
$$

Then $e_{0}, e_{1}, \ldots, e_{r},-e^{0}, e^{1}, \ldots, e^{r}$ form a symplectic basis for $Q$.

(3A) A-model connection $\nabla=\nabla^{\text {middle }}$ of $V$.

For $\delta_{j}:=\partial / \partial u_{j}$, A-model connection is defined by

$$
\begin{aligned}
& \nabla_{\delta_{j}} T^{0}:=0, \quad \nabla_{\delta_{j}} T^{k}:=\delta_{j k} T^{0} \\
& \nabla_{\delta_{j}} T_{k}:=\frac{1}{(2 \pi i)^{3}} \sum_{l} \frac{\partial^{3} \Phi}{\partial u_{j} \partial u_{k} \partial u_{l}} T^{l}, \\
& \nabla_{\delta_{j}} T_{0}:=T_{j},
\end{aligned}
$$

where the indices are $1 \leq j, k, l \leq r$. Note that $\nabla^{2}=0$ holds.

(3B) B-model connection $\nabla=\nabla^{\mathrm{GM}}$ of $V^{\circ}$.

For $\delta_{j}:=\partial / \partial u_{j}$, the Gauss-Manin connection is computed as

$$
\begin{aligned}
& \nabla_{\delta_{j}} e^{0}:=0, \quad \nabla_{\delta_{j}} e^{k}:=\delta_{j k} T^{0}, \\
& \nabla_{\delta_{j}} e_{k}:=\frac{1}{(2 \pi i)^{3}} \sum_{l} \frac{\partial^{3} \Phi}{\partial u_{j} \partial u_{k} \partial u_{l}} e^{l}=\sum_{l} Y_{j k l} e^{l}, \\
& \nabla_{\delta_{j}} e_{0}:=e_{j},
\end{aligned}
$$

where the indices are $1 \leq j, k, l \leq r$ (cf. [CK99, Formula (5.67)]).

(4A) $\nabla$-flat $\mathbf{Z}$-basis for $\mathcal{H}_{\mathbf{Z}}^{V}$

First, we compute $\nabla$-flat elements of $\mathcal{O}_{S, b}^{\log } \otimes W_{2 p}$ whose image in $\operatorname{gr}_{2 p}^{W}$ belong to 15 
$H^{3-p, 3-p}(V, \mathbf{Z})$.

$$
\begin{aligned}
& \tilde{s}^{0}:=T^{0}, \\
& \tilde{s}^{k}:=T^{k}-u_{k} T^{0} \quad(1 \leq k \leq r), \\
& \tilde{s}_{k}:=T_{k}-\frac{1}{(2 \pi i)^{3}} \sum_{l} \frac{\partial^{2} \Phi}{\partial u_{k} \partial u_{l}} T^{l}+\frac{1}{(2 \pi i)^{3}} \frac{\partial \Phi}{\partial u_{k}} T^{0} \\
& =T_{k}-\sum_{j, l}\left(\int_{V} T_{k} \cup T_{j} \cup T_{l}\right) u_{j} T^{l}+\frac{1}{2} \sum_{j, l}\left(\int_{V} T_{k} \cup T_{j} \cup T_{l}\right) u_{j} u_{l} T^{0} \\
& -\left(\sum_{\beta} \frac{N_{\beta}\left(\int_{\beta} T_{k}\right)\left(\int_{\beta} T_{l}\right)}{2 \pi i} q^{\beta}\right) T^{l}+\left(\sum_{\beta} \frac{N_{\beta}\left(\int_{\beta} T_{k}\right)}{(2 \pi i)^{2}} q^{\beta}\right) T^{0} \quad(1 \leq k \leq r), \\
& \tilde{s}_{0}:=T_{0}-\sum_{l} u_{l} T_{l}+\frac{1}{(2 \pi i)^{3}} \sum_{l}\left(\sum_{j} u_{j} \frac{\partial^{2} \Phi}{\partial u_{j} \partial u_{l}}-\frac{\partial \Phi}{\partial u_{l}}\right) T^{l} \\
& -\frac{1}{(2 \pi i)^{3}}\left(\sum_{j} u_{j} \frac{\partial \Phi}{\partial u_{j}}-2 \Phi\right) T^{0} \\
& =T_{0}-\sum_{j} u_{j} T_{j}+\frac{1}{2} \sum_{j, k, l}\left(\int_{V} T_{j} \cup T_{k} \cup T_{l}\right) u_{j} u_{k} T^{l} \\
& -\frac{1}{6} \sum_{j, k, l}\left(\int_{V} T_{j} \cup T_{k} \cup T_{l}\right) u_{j} u_{k} u_{l} T^{0} \\
& +\sum_{l}\left(\sum_{j} \sum_{\beta} \frac{N_{\beta}\left(\int_{\beta} T_{j}\right)\left(\int_{\beta} T_{l}\right)}{2 \pi i} u_{j} q^{\beta}-\sum_{\beta} \frac{N_{\beta}\left(\int_{\beta} T_{l}\right)}{(2 \pi i)^{2}} q^{\beta}\right) T^{l} \\
& -\left(\sum_{j} \sum_{\beta} \frac{N_{\beta}\left(\int_{\beta} T_{j}\right)}{(2 \pi i)^{2}} u_{j} q^{\beta}-\sum_{\beta} \frac{2 N_{\beta}}{(2 \pi i)^{3}} q^{\beta}\right) T^{0} .
\end{aligned}
$$

Find $c^{k 0}, c_{k}^{j}, c_{k}^{0}, c_{0 j}, c_{0}^{j}, c_{0}^{0} \in \mathbf{C}(1 \leq j, k \leq r)$ such that

$$
\begin{aligned}
& \tilde{s}^{0}\left(u_{0}\right)=b^{0} \\
& \tilde{s}^{k}\left(u_{0}\right)=b^{k}+c^{k 0} \tilde{s}^{0}\left(u_{0}\right) \quad(1 \leq k \leq r), \\
& \tilde{s}_{k}\left(u_{0}\right)=b_{k}+\sum_{j} c_{k}^{j} \tilde{s}^{j}\left(u_{0}\right)+c_{k}^{0} \tilde{s}^{0}\left(u_{0}\right) \quad(1 \leq k \leq r), \\
& \tilde{s}_{0}\left(u_{0}\right)=b_{0}+\sum_{j} c_{0 j} \tilde{s}_{j}\left(u_{0}\right)+\sum_{j} c_{0}^{j} \tilde{s}^{j}\left(u_{0}\right)+c_{0}^{0} \tilde{s}^{0}\left(u_{0}\right),
\end{aligned}
$$


then we have the desired basis:

$$
\begin{aligned}
& s^{0}:=\tilde{s}^{0}, \\
& s^{k}:=\tilde{s}^{k}-c^{k 0} \tilde{s}^{0}\left(u_{0}\right) \quad(1 \leq k \leq r), \\
& s_{k}:=\tilde{s}_{k}-\sum_{j} c_{k}^{j} \tilde{s}^{j}-c_{k}^{0} \tilde{s}^{0} \quad(1 \leq k \leq r), \\
& s_{0}:=\tilde{s}_{0}-\sum_{j} c_{0 j} \tilde{s}_{j}-\sum_{j} c_{0}^{j} \tilde{s}^{j}-c_{0}^{0} \tilde{s}^{0},
\end{aligned}
$$

that is,

$$
\begin{aligned}
& s^{0}:= T^{0}, \\
& s^{k}:=T^{k}-\left(u_{k}+c^{k 0}\right) T^{0}, \\
& s_{k}:=T_{k}-\left(\sum_{j} \frac{1}{(2 \pi i)^{3}} \frac{\partial^{2} \Phi}{\partial u_{k} \partial u_{j}}+c_{k}^{j}\right) T^{j}+\left(\frac{1}{(2 \pi i)^{3}} \frac{\partial \Phi}{\partial u_{k}}+\sum_{j} c_{k}^{j} u_{j}-c_{k}^{0}\right) T^{0}, \\
& s_{0}:=T_{0}-\sum_{j}\left(u_{j}+c_{0 j}\right) T_{j}+\left(\sum_{l} \frac{1}{(2 \pi i)^{3}}\left(\sum_{j}\left(u_{j}+c_{0 j}\right) \frac{\partial^{2} \Phi}{\partial u_{j} \partial u_{l}}-\frac{\partial \Phi}{\partial u_{l}}\right)-c_{0}^{l}\right) T^{l} \\
&-\left(\frac{1}{(2 \pi i)^{3}}\left(\sum_{j}\left(u_{j}+c_{0 j}\right) \frac{\partial \Phi}{\partial u_{j}}-2 \Phi\right)-c_{0}^{j} u_{j}+c_{0}^{0}\right) T^{0} .
\end{aligned}
$$

(4B) $\nabla$-flat $\mathbf{Z}$-basis for $\mathcal{H}_{\mathbf{Z}}^{V^{\circ}}$.

Similarly, it is computed as

$$
\begin{aligned}
& s^{0}:=e^{0}, \\
& s^{k}:=e^{k}-\left(u_{k}+c^{k 0}\right) e^{0}, \\
& s_{k}:=e_{k}-\left(\sum_{j} \frac{1}{(2 \pi i)^{3}} \frac{\partial^{2} \Phi}{\partial u_{k} \partial u_{j}}+c_{k}^{j}\right) e^{j}+\left(\frac{1}{(2 \pi i)^{3}} \frac{\partial \Phi}{\partial u_{k}}+\sum_{j} c_{k}^{j} u_{j}-c_{k}^{0}\right) e^{0}, \\
& s_{0}:=e_{0}-\sum_{j}\left(u_{j}+c_{0 j}\right) e_{j}+\left(\sum_{l} \frac{1}{(2 \pi i)^{3}}\left(\sum_{j}\left(u_{j}+c_{0 j}\right) \frac{\partial^{2} \Phi}{\partial u_{j} \partial u_{l}}-\frac{\partial \Phi}{\partial u_{l}}\right)-c_{0}^{l}\right) e^{l} \\
&-\left(\frac{1}{(2 \pi i)^{3}}\left(\sum_{j}\left(u_{j}+c_{0 j}\right) \frac{\partial \Phi}{\partial u_{j}}-2 \Phi\right)-c_{0}^{j} u_{j}+c_{0}^{0}\right) e^{0} .
\end{aligned}
$$

(5A) Expression of the $T^{i}, T_{i}$ by the $s^{i}, s_{i}$. 
It is computed that $T^{i}, T_{i}$ are written by the $\nabla$-flat $\mathbf{Z}$-basis $s^{i}, s_{i}$ of $\mathcal{H}_{\mathbf{Z}}^{V}$ as follows.

$$
\begin{aligned}
T^{0}= & s^{0} \\
T^{k}= & s^{k}+\left(u_{k}+c^{k 0}\right) s^{0}, \\
T_{k}:= & s_{k}+\left(\sum_{j} \frac{1}{(2 \pi i)^{3}} \frac{\partial^{2} \Phi}{\partial u_{k} \partial u_{j}}+c_{k}^{j}\right) s^{j} \\
& +\left(\frac{1}{(2 \pi i)^{3}}\left(\sum_{j}\left(u_{j}+c^{j 0}\right) \frac{\partial^{2} \Phi}{\partial u_{k} \partial u_{j}}-\frac{\partial \Phi}{\partial u_{k}}\right)+\sum_{j} c_{k}^{j} c^{j 0}+c_{k}^{0}\right) s^{0}, \\
T_{0}= & s_{0}+\sum_{j}\left(u_{j}+c_{0 j}\right) s_{j}+\sum_{j}\left(\frac{1}{(2 \pi i)^{3}} \frac{\partial \Phi}{\partial u_{j}}+\sum_{k}\left(u_{k}+c_{0 k}\right) c_{k}^{j}+c_{0}^{j}\right) s^{j} \\
& +\left(\frac{1}{(2 \pi i)^{3}}\left(\sum_{j}\left(u_{j}+c^{j 0}\right) \frac{\partial \Phi}{\partial u_{j}}-2 \Phi\right)+\sum_{j}\left(u_{j}+c_{0 j}\right)\left(\sum_{k} c_{j}^{k} c^{k 0}+c_{j}^{0}\right)\right. \\
& \left.+\sum_{j} c_{0}^{j} c^{j 0}+c_{0}^{0}\right) s^{0}
\end{aligned}
$$

Note that the section $1=T_{0}$ varies with respect to the the lattice $\mathcal{H}_{\mathbf{Z}}^{V}$ as above while the section $[\mathrm{pt}]=T^{0}=s^{0}$ does not.

(5B) Expression of the $e^{i}, e_{i}$ by the $s^{i}, s_{i}$.

It is computed that $e^{i}, e_{i}$ are written by the $\nabla$-flat $\mathbf{Z}$-basis $s^{i}, s_{i}$ of $\mathcal{H}_{\mathbf{Z}}^{V^{\circ}}$ as follows.

$$
\begin{aligned}
e^{0}= & s^{0} \\
e^{k}= & s^{k}+\left(u_{k}+c^{k 0}\right) s^{0}, \\
e_{k}:= & s_{k}+\left(\sum_{j} \frac{1}{(2 \pi i)^{3}} \frac{\partial^{2} \Phi}{\partial u_{k} \partial u_{j}}+c_{k}^{j}\right) s^{j} \\
& +\left(\frac{1}{(2 \pi i)^{3}}\left(\sum_{j}\left(u_{j}+c^{j 0}\right) \frac{\partial^{2} \Phi}{\partial u_{k} \partial u_{j}}-\frac{\partial \Phi}{\partial u_{k}}\right)+\sum_{j} c_{k}^{j} c^{j 0}+c_{k}^{0}\right) s^{0}, \\
e_{0}= & s_{0}+\sum_{j}\left(u_{j}+c_{0 j}\right) s_{j}+\sum_{j}\left(\frac{1}{(2 \pi i)^{3}} \frac{\partial \Phi}{\partial u_{j}}+\sum_{k}\left(u_{k}+c_{0 k}\right) c_{k}^{j}+c_{0}^{j}\right) s^{j} \\
& +\left(\frac{1}{(2 \pi i)^{3}}\left(\sum_{j}\left(u_{j}+c^{j 0}\right) \frac{\partial \Phi}{\partial u_{j}}-2 \Phi\right)+\sum_{j}\left(u_{j}+c_{0 j}\right)\left(\sum_{k} c_{j}^{k} c^{k 0}+c_{j}^{0}\right)\right. \\
& \left.+\sum_{j} c_{0}^{j} c^{j 0}+c_{0}^{0}\right) s^{0}
\end{aligned}
$$

Note that the normalized holomorphic 3 -form $\tilde{\Omega}:=\Omega / y_{0}=e_{0}$ varies with respect to the lattice $\mathcal{H}_{\mathbf{Z}}^{V^{\circ}}$ as above, while the section $g_{0}=e^{0}=s^{0}$ does not.

(7A) A-model monodromy weight filtration of $\mathcal{H}^{V}$. 
It is computed as follows, which does not vary.

$$
\begin{aligned}
& W_{0}=\mathbf{R}[\mathrm{pt}]=\mathbf{R} T^{0}=\mathbf{R} s^{0}=W_{1} \subset W_{2}=W_{1}+\sum_{j=1}^{r} \mathbf{R} T^{j}=W_{3} \\
& \subset W_{4}=W_{3}+\sum_{j=1}^{r} \mathbf{R} T_{j}=W_{5} \subset W_{6}=W_{5}+\mathbf{R} \cdot 1=W_{5}+\mathbf{R} T_{0} .
\end{aligned}
$$

(8A) A-model Hodge filtration of $\mathcal{H}^{V}$.

Let $\mathcal{F}^{p}:=F^{p} \times \overline{\mathcal{K} \mathcal{M}}(V) \subset \mathcal{H}_{\mathcal{O}}^{V}$. This is computed as follows.

$$
\begin{aligned}
& \mathcal{F}^{3}=H^{0,0}(V)=\mathbf{C} \cdot 1=\mathbf{C} T_{0} \subset \mathcal{F}^{2}=\mathcal{F}^{3}+H^{1,1}(V)=\mathcal{F}^{3}+\sum_{j=1}^{r} \mathbf{C} T_{j} \\
& \subset \mathcal{F}^{1}=\mathcal{F}^{2}+H^{2,2}(V)=\mathcal{F}^{2}+\sum_{j=1}^{r} \mathbf{C} T^{j} \\
& \subset \mathcal{F}^{0}=\mathcal{F}^{1}+H^{3,3}(V)=\mathcal{F}^{1}+\mathbf{C}[\mathrm{pt}]=\mathcal{F}^{1}+\mathbf{C} T^{0} .
\end{aligned}
$$

Note that the mixed Hodge structure for A-model of $V$ from (7A) and (8A) is opposite to the original one from $(2 \mathrm{~A})$.

\subsection{On equivalence of formulations (1)-(4) in Introduction}

The equivalence of the formulations (1)-(3) in Introduction are shown in [CK99, Section 8.6]. We prove here the equivalence of (3) and (4) in Introduction. We give two proofs. One is an application of the log Hodge theoretic interpretation of the nilpotent orbit theorem, and the other is an elementary direct computation by using the table in 2.5 .

Proof 1 , by nilpotent orbit theorem. We show $(3) \Rightarrow(4)$ in Introduction. Let $S^{*}:=$ $\mathcal{K} \mathcal{M}(V)$ and $S:=\overline{\mathcal{K} \mathcal{M}}(V)$ for A-model, and $S^{*}:=\mathcal{M}\left(V^{\circ}\right)$ and $S:=\overline{\mathcal{M}}\left(V^{\circ}\right)$ for Bmodel. $S$ is endowed with the log structure associated to the normal crossing divisor $S \backslash S^{*}$. In both cases, the variation of Hodge structure on $S^{*}$ extend to a log Hodge structure on $S$ by the log Hodge theoretic interpretation [KU09, Theorem 2.5.14] of the nilpotent orbit theorem of Schmid [S73]. The specified sections $1=T_{0}$ for A-model and $\tilde{\Omega}=e_{0}$ for B-model extends uniquely over $S$ as the canonical extensions, and the specified sections $[\mathrm{pt}]=T^{0}$ for A-model and $g_{0}:=e^{0}$ for B-model extend over $S$ as invariant sections, and obviously they coincide respectively.

The converse implication $(4) \Rightarrow(3)$ in Introduction is just the restriction.

Proof 2, by correspondence table in 2.5. We use the correspondence table in 2.5. The implication $(3) \Rightarrow(4)$ in Introduction is verified directly as follows.

The local system $H_{\mathbf{Z}}:=\mathcal{H}_{\mathbf{Z}}^{V}$ for A-model and $\mathcal{H}_{\mathbf{Z}}^{V^{\circ}}$ for B-model over $S^{*}$, endowed with the respective symplectic basis $s_{0}, s_{1}, \ldots, s_{r},-s^{0}, s^{1}, \ldots, s^{r}$ from (4A) and (4B) in 2.5 , extends as a local system over $S^{\log }$ with the extended symplectic basis ([O03], cf. [KU09, Proposition 2.3.5]).

We use the base points $u_{0}=(0+i \infty)^{r} \in \tilde{S}^{\log } \mapsto b:=(\overline{0}+i \infty)^{r} \in S^{\log } \mapsto q=$

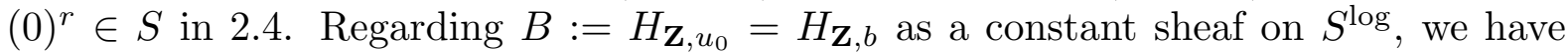
an isomorphism $\mathcal{O}_{S}^{\log } \otimes H_{\mathbf{Z}} \simeq \mathcal{O}_{S}^{\log } \otimes B$ of $\mathcal{O}_{S}^{\log }$-modules whose restriction induces the 
identity $1 \otimes H_{\mathbf{Z}, b}=1 \otimes B$ ([KU09, Proposition 2.3.2], cf. A list of errata loc.cit. on a web page of press.princeton.edu.).

We explain the following: The direct image $\tau_{*}\left(\mathcal{O}_{S}^{\log } \otimes H_{\mathbf{Z}}\right)$ yields the Deligne canonical extension of $H_{\mathcal{O}_{S^{*}}}$ over $S$. The bases $T_{0}, T_{1}, \ldots, T_{r}, T^{1}, \ldots, T^{r}, T^{0}$ from (2A) and $e_{0}, e_{1}, \ldots, e_{r}, e^{1}, \ldots, e^{r}, e^{0}$ from $(2 \mathrm{~B})$ in 2.5 yield monodromy invariant bases of $\mathcal{O}_{S^{*}-}$ modules respecting Hodge filtration for each case. These bases and hence Hodge filtrations extend over the boundary $\prod_{j=1}^{r} q_{j}=0$.

This follows from the following observations (a)-(c). Denote by $R$ the $\operatorname{ring} \mathcal{O}_{S, 0}^{\log }=$ $\mathbf{C}\left\{q_{1}, \ldots, q_{r}\right\}\left[u_{1}, \ldots, u_{r}\right]$, where $u_{1}, \ldots, u_{r}$ from 2.3 and 2.2 are considered as multivalued functions on a neighborhood of $0 \in S$ in $S^{*}$.

(a) $T_{j}, T^{j}$ and $e_{j}, e^{j}$ are $R$-linear combinations of respective $s_{j}, s^{j}$.

(b) $s_{j}, s^{j}$ are $R$-linear combinations of $s_{j}(b), s^{j}(b) \in H_{\mathbf{Z}, b}=B$.

(c) The coefficients $h \in R$ of the composition of (a) and (b) are monodromy invariant holomorphic functions on $S^{*}$ which are locally bounded on $S$. Hence the divisor $\prod_{j} q_{j}=$ 0 is removable singularities of $h$. Thus $h$ extends uniquely as a holomorphic function on $S$.

(Using the table in 2.5, we can also examine the above argument by a direct computation.)

Thus, the variation of polarized Hodge structure $\left(H_{\mathbf{Z}},\langle\rangle,, H_{\mathcal{O}}\right)$ of type $(\Lambda, \Gamma)$ over $S^{*}$ extends to a pre-PLH of type $\Phi=(\Lambda, \sigma, \Gamma)$ over $S$, where $\Gamma$ and $\sigma$ are from (4) in Introduction. (Note that $\sigma$ here is $-\sigma$ of the $\sigma$ in Section 1.)

Now we verify that this pre-PLH is indeed a PLH. The admissibility is obvious in the pure case. The big Griffiths transversality 1.3 (3) follows from the above choice of the bases $T_{0}, T_{1}, \ldots, T_{r}, T^{1}, \ldots, T^{r}, T^{0}$ and $e_{0}, e_{1}, \ldots, e_{r}, e^{1}, \ldots, e^{r}, e^{0}$. The positivity 1.3 (2) is verified by a direct computation using these bases and the polarizations $S$ from (1A) and $Q$ from (1B) in 2.5 in the following way. We do this for B-model case. A-model case is analogous. Let $F(0) \in \check{D}$ be the fixed Hodge filtration determined by the basis $e_{0}\left(u_{0}\right), e_{1}\left(u_{0}\right), \ldots, e_{r}\left(u_{0}\right), e^{1}\left(u_{0}\right), \ldots, e^{r}\left(u_{0}\right), e^{0}\left(u_{0}\right)$. Let $F_{\eta}:=\exp \left(\sum_{j} i \eta_{j}\left(-N_{j}\right)\right) F(0)$. Then, $v_{0}(\eta):=\exp \left(\sum_{j} i \eta_{j}\left(-N_{j}\right)\right) e_{0}\left(u_{0}\right), \ldots, \exp \left(\sum_{j} i \eta_{j}\left(-N_{j}\right)\right) e_{r}\left(u_{0}\right)$ is a basis of $F_{\eta}^{2}$ respecting $F_{\eta}^{3}$. From this, we can compute a basis $v_{j}(\eta)(1 \leq j \leq r)$ of $F_{\eta}^{2} \cap \overline{F_{\eta}^{1}}=$ $F_{\eta}^{2} \cap\left(\overline{F_{\eta}^{3}}\right)^{\perp}$, where ${ }^{\perp}$ is with respect to $Q$. We can check that the coefficients of the highest terms in the total degree in the variables $\eta_{j}$ of the Hodge norms $i^{3} Q\left(v_{0}(\eta), \overline{v_{0}(\eta)}\right)$ and $i Q\left(\sum_{j=1}^{r} a_{j} v_{j}(\eta), \overline{\sum_{j=1}^{r} a_{j} v_{j}(\eta)}\right)\left(a_{1}, \ldots, a_{r} \in \mathbf{C}\right)$ are both positive. This nilpotent $i$-orbit and the original Hodge filtration corresponding to $e_{0}, e_{1}, \ldots, e_{r}, e^{1}, \ldots, e^{r}, e^{0}$ are asymptotic. The positivity follows.

The above result is interpreted into log period map by 1.6 .

\section{REFERENCES}

[CK99] D. A. Cox and S. Katz, Mirror symmetry and algebraic geometry, Math. Surveys and Monographs, vol. 68, AMS, 1999, pp. 469.

[D97] P. Deligne, Local behavior of Hodge structures at infinity, in Mirror Symmetry II (B. Greene and S.-T. Yau, eds.), AMS/IP Stud. Adv. Math. 1, 1997, 683-699. 
[G68] P. A. Griffiths, Periods of integrals on algebraic manifolds, I. Construction and properties of the modular varieties, Amer. J. Math. 90 (1968), 568-626.

[I11] H. Iritani, Quantum cohomology and periods, Ann. Inst. Fourier 61-7 (2011), 2909-2958.

[KNU08] K. Kato, C. Nakayama and S. Usui, SL(2)-orbit theorem for degeneration of mixed Hodge structure, J. Algebraic Geometry 17 (2008), 401-479.

[KNU09] _ Classifying spaces of degenerating mixed Hodge structures, I: Borel-Serre spaces, Advanced Studies in Pure Math. 54: Algebraic Analysis and Around, 2009, 187-222.

[KNU11] _ Classifying spaces of degenerating mixed Hodge structures, II: Spaces of SL(2)orbits, to appear in Kyoto J. Math. 51-1: Nagata Memorial Issue (2011), 149-261. (available in arXiv).

[KNU13] _ Classifying spaces of degenerating mixed Hodge structures, III: Spaces of nilpotent orbits, J. Algebraic Geometry, 22 (2013), 671-772.

[KNU14] _ Néron models for admissible normal functions, Proc. Japan Acad. 90, Ser. A No. 1 (2014), 6-10.

[KU99] K. Kato and S. Usui, Logarithmic Hodge structures and classifying spaces (summary), in CRM Proc. \& Lect. Notes: The Arithmetic and Geometry of Algebraic Cycles, (NATO Advanced Study Institute / CRM Summer School 1998: Banff, Canada) 24 (1999), 115130.

[KU02] _ Borel-Serre spaces and spaces of SL(2)-orbits, Advanced Studies in Pure Math. 36: Algebraic Geometry 2000, Azumino, (2002), 321-382.

[KU09] _ Classifying spaces of degenerating polarized Hodge structures, Ann. Math. Studies, Princeton Univ. Press, (A list of errata is on the web page of ), vol. 169, Princeton, 2009, pp. 288. (A list of errata is on a web page of press.princeton.edu).

[M93] D. Morrison, Compactifications of moduli spaces inspired by mirror symmetry, in Journées de Géométrie Algébrique d'Orsay (Orsay, 1992), Astérisque 218 (1993), 243-271.

[M97] _ Mathematical aspects of mirror symmetry, in Complex algebraic geometry (Park City, UT, 1993), IAS/Park City Math. Ser. 3, AMS (1997), 265-327.

[S73] W. Schmid, Variation of Hodge structure: The singularities of the period mapping, Invent. Math. 22 (1973), 211-319.

[U13p] S. Usui, A study of mirror symmetry through log mixed Hodge theory, Cont. Math., AMS (to appear).

[U14p] _ Studies of closed/open mirror symmetry for quintic three-folds through log mixed Hodge theory (in preparation).

Sampei USUI

Graduate School of Science

Osaka University

Toyonaka, Osaka, 560-0043, Japan

usui@math.sci.osaka-u.ac.jp 